\title{
ON THE CLASS NUMBERS OF A CYCLIC FIELD AND A SUB-FIELD*
}

\author{
C. G. LATIMER
}

1. Introduction. Let $F_{1}$ be an algebraic field which is cyclic with respect to the rational field and let $F_{2}$ be a sub-field of $F_{1}$. Kummer stated that if $F_{1}$ is a divisor of the field defined by a $\lambda$ th root of unity, $\lambda$ a prime, then the class number of $F_{2}$ is a divisor of the class number of $F_{1} \dagger$ He employed the broader definition of equivalence. However, as pointed out by Hilbert, $\ddagger$ there is an error in his proof. Furtwängler proved the theorem for the case where $\lambda$ is a power of a prime, using narrow equivalence. $\S$

The purpose of this paper is to prove the following theorem which overlaps but does not include Furtwängler's.

THEOREM. Let $F_{1}$ be a field which is cyclic with respect to the rational field and such that the discriminant of every sub-field, not rational, contains a prime factor not a divisor of the degree $F_{1}$. If $F_{2}$ is a sub-field of $F_{1}$ and if $h_{1}, h_{2}$ are the number of classes of narrowly equivalent ideals in $F_{1}, F_{2}$, respectively, then $h_{2}$ is a divisor of $h_{1}$.

Furtwängler gave an example of a non-cyclic abelian field $F_{1}$, for which this theorem is not valid.||

2. A Lemma. In the next paragraph, the above theorem will be proved by use of the following lemma and a theorem due to Chevally.

* Presented to the Society, December 27, 1932.

$\dagger$ Journal für Mathematik, vol. 40 (1850), pp. 114-6; Bulletin of the National Research Council, No. 62, Algebraic Numbers, II, Vandiver and Wahlin, p. 16.

$\ddagger$ Bericht über die Theorie der algebraischen Zahlkörper, p. 378.

§ Journal für Mathematik, vol. 134 (1908), pp. 91-94. In this article, Furtwängler states (p. 91) that Kummer's theorem is correct since his result is a generalization of Kummer's. Since he and Kummer used different definitions of equivalence, it is not obvious that his theorem includes Kummer's and the validity of the latter is still an open question.

|| Loc. cit., p. 94. 
Lemma. Let $K$ be a field such that $F_{1} \geqq K>F_{2}$. If $K$ is of degree $e$ with respect to a sub-field $F$, where $e$ is a prime, and if the discriminants of $K$ and $F$ are $D$ and $d$ respectively, then $D \neq d$.

Every prime factor of $d$ is a factor of $D$. If $D$ contains a prime factor not a divisor of $d$, the lemma follows at once. We shall therefore assume hereafter that a prime divides $D$ only if it divides $d$.

$F_{1}$ is cyclic and hence the same is true of $K$ and $F$. Let $F$ be of degree $E=e_{1} \cdot e_{2} \cdots e_{m}$, where the $e^{\prime}$ s are powers of distinct primes. Since $F$ is an abelian field, it may be obtained by composition of cyclic abelian fields of prime power degree and these prime powers are the invariants of the Galois group of $F{ }^{*}$ Since this group is cyclic, these invariants are relatively prime in pairs. It follows that $e_{1}, e_{2}, \cdots, e_{m}$ are the invariants of the group and $F$ is obtained by composition of $m$ cyclic fields $F^{\left(e_{1}\right)}$, $F^{\left(e_{2}\right)}, \cdots, F^{\left(e_{m}\right)}$ of degrees $e_{1}, e_{2}, \cdots, e_{m}$, respectively.

The degree $e E$ of $K$ is a divisor of the degree of $F_{1}$ and therefore, by the hypothesis of the theorem, $D$ contains a prime factor $p$, not a divisor of $e E$. Then $p$ is a divisor of $d$ and hence it is a divisor of the discriminant of at least one of the fields $F^{\left(e_{i}\right)}$. After proper choice of notation, we may assume that $p$ is a divisor of the discriminant of each of the fields $F^{\left(e_{i}\right)},(i=1,2$, $\cdots, s \leqq m$ ), and, if $s<m$, it is not a divisor of the discriminant of any of the fields $F^{\left(e_{i}\right)},(i=s+1, \cdots, m)$.

Since every $F^{\left(e_{i}\right)}$ is a cyclic field of prime power degree, it follows that

$$
p=\mathfrak{B}_{i}{ }^{{ }^{i} i}, \quad(i=1,2, \cdots, s),
$$

where $\mathfrak{P}_{i}$ is a prime ideal in $F^{\left(e_{i}\right)}, \dagger$ Employing the fact that the $e_{i}$ are relatively prime in pairs, we may show that if $F^{\left(E_{1}\right)}$ is the field of degree $E_{1}=e_{1} \cdot e_{2} \cdots e_{s}$ obtained by composition of the $F^{\left(e_{i}\right)},(i=1,2, \cdots, s)$, then

$$
p=\mathfrak{P}^{\prime E_{1}},
$$

where $\mathfrak{B}^{\prime}$ is a prime ideal in the field $F^{\left(E_{1}\right)}$. If $E=E_{1}, d$ is divisible by $p^{E-1}$ and by no higher power of $p . \ddagger$ Suppose $E / E_{1}$

* Weber, Lehrbuch der Algebra, 2d edition, vol. 2, p. 764.

$\dagger$ Weber, Zur Theorie der zyklischen Zahlkörper, Mathematische Annalen, vol. 67 (1909), p. 48.

$\ddagger$ Bachmann, Allgemeine Arithmetik der Zahlenkörper, p. 294. 
$=E_{2}>1$. Let $F^{\left(E_{2}\right)}$ be the field obtained by composition of the fields $F^{\left(e_{i}\right)},(i=s+1, \cdots, m)$. Since $p$ is not a divisor of the discriminant of any of these $m-s$ fields, it is not a divisor of the discriminant of $F^{\left(E_{2}\right)}$. Hence

$$
p=\mathfrak{P}_{1}^{\prime \prime} \cdot \mathfrak{P}_{2}^{\prime \prime} \cdot \cdot \mathfrak{B}_{k}^{\prime \prime},
$$

where the $\mathfrak{B}_{i}{ }^{\prime \prime}$ are distinct prime ideals in $F^{\left(E_{2}\right)}$, each of degree $t=E_{2} / k$. The field $F$ is obtained by composition of $F^{\left(E_{1}\right)}, F^{\left(E_{2}\right)}$. Employing the above factorizations of $p$ in these fields, it may be shown that

$$
p=\left(\mathfrak{p}_{1} \cdot \mathfrak{p}_{2} \cdots \mathfrak{p}_{k}\right)^{E_{1}}
$$

where the $\mathfrak{p}_{i}$ are distinct prime ideals in $F$, each of degree $t$. It follows that

$$
d=p^{E_{2}\left(E_{1}-1\right)} \cdot d_{1}=p^{E\left(1-E_{1}^{-1}\right)} \cdot d_{1},
$$

where $d_{1}$ is prime to $p . *$ This holds if $E=E_{1}$.

Consider the field $K$. It is of degree $e E$. If $e$ is prime to $E$, it may be shown, as for $F$, that $K$ is obtained by composition of the above fields $F^{\left(e_{i}\right)}$, and another cyclic field $F^{(e)}$ of degree $e$. If $e$ is not prime to $E$, it divides one of the $e_{i}$, say $e_{1}$. Then it may be shown that $K$ is obtained by composition of a cyclic field $F^{\left(e e_{1}\right)}$ of degree $e e_{1}$, and the fields $F^{\left(e_{2}\right)}, F^{\left(e_{3}\right)}, \cdots, F^{\left(e_{m}\right)}$.

We have assumed before that $p$ is a divisor of $D$. By the condition of the theorem on the sub-fields of $F_{1}$, we may assume that it is also a divisor of the discriminant of $F^{(e)}$, or $F^{\left(e e_{1}\right)}$. We find, as for $F$, that

$$
D=p^{e E\left(1-E_{1}^{\prime-1}\right)} \cdot D_{1}
$$

where $D_{1}$ is prime to $p$ and $E_{1}{ }^{\prime}$ is the product of the degrees of those fields $F^{(e)}, F^{\left(e_{1}\right)}, \cdots, F^{\left(e_{m}\right)}$ or $F^{\left(e e_{1}\right)}, F^{\left(e_{2}\right)}, \cdots, F^{\left(e_{m}\right)}$ whose discriminants are divisible by $p$. Since one of these fields is $F^{(e)}$, or $F^{\left(e e_{1}\right)}$, it follows that $E_{1}{ }^{\prime} \neq E_{1}$. The lemma follows from the above expressions for $d$ and $D$.

3. Proof of Theorem. Let $K$ be a field such that $F_{1} \geqq K>F_{2}$. There is a sequence of fields $K=K_{0}>K_{1}>\cdots>K_{t}=F_{2}$ such that the degree of every $K_{i-1}$ with respect to $K_{i}$ is a prime $e^{(i)}$. If $D_{j}$ is the discriminant of $K_{j}$, then

$$
D_{i-1}=D_{i} e^{(i)} N\left(\mathfrak{D}_{i}\right), \quad(i=1,2, \cdots, t),
$$

\footnotetext{
* Bachmann, loc. cit.
} 
where $\mathfrak{D}_{i}$ is* the relative discriminant of $K_{i-1}$ with respect to $K_{i}$. The field $K$ is of degree $\epsilon=e^{(1)} \cdot e^{(2)} \cdots e^{(t)}$ with respect to $F_{2}$ and by the last reference

$$
D=d^{\epsilon} N(\mathfrak{D}),
$$

where $\mathfrak{D}$ is the relative discriminant of $K$ with respect to $F_{2}$. By the Lemma, every $N\left(\mathfrak{D}_{i}\right)>1$. It follows that $N(\mathfrak{D})>1$. But, by a result due to Chevally, $h_{2}$ divides $h_{1}$ if there is no field $K$, $F_{1} \geqq K>F_{1}$, such that the relative discriminant of $K$ with respect to $F_{2}$ is of norm unity. $\dagger$ The theorem follows.

University of Kentucky

\section{SOME APPLICATIONS OF MURPHY'S THEOREM $\ddagger$}

\section{BY H. BATEMAN}

It is well known that the linear partial differential equations of mathematical physics possess solutions in the form of definite integrals with limits depending on the variables entering into the partial differential equations. The law connecting the limits of such an integral with the integrand looks at first sight rather mysterious but the whole matter becomes clear when the integral is expressed as a contour integral with the aid of a theorem due to Murphy and, in a slightly different form, to Cauchy. $\S$

If $C$ is a closed contour containing just one root, $a$, of the equation $F(x)=0$ and just one root, $b$, of the equation $G(x)=0$, then, if the radii from these roots turn completely round just once and in one direction as a point describes this contour and if the functions $f(z), \int f(z) d z, F(z)$, and $G(z)$ are analytic and uni-

* Bachmann, loc. cit., p. 452.

$\dagger$ Chevally, Relation entre le nombre de classes d'un sous-corps et celui d'un sur-corps, Comptes Rendus, vol. 192 (1931), pp. 257-258.

$\ddagger$ Presented to the Society, December 27, 1932.

$\S$ R. Murphy, Transactions of the Cambridge Philosophical Society, vol. 3 (1830), p. 429, A. L. Cauchy, Journal de l'École Polytechnique, vol. 12 (1823), p. 580. Murphy's integral has been transformed into a contour integral from which Cauchy's relation may be obtained by an integration by parts. 